\title{
Flu and Flu Vaccination in Comparison, in the Evaluation of the Health Status of Children
}

\author{
Gaetano Bottaro ${ }^{1,}$, Giuseppe Bottaro ${ }^{2}$, Filippo Palermo ${ }^{3}$ \\ ${ }^{1}$ Italian National Health System, Catania Health Authority, Department of Community Medicine, Catania, Italy \\ ${ }^{2}$ Department of Cardiology, Policlinico-Vittorio Emanuele Hospital, University of Catania, Catania, Italy \\ ${ }^{3}$ Department of Medicine, Catania University, Catania, Italy \\ Email address: \\ gaetano.bottaro@tiscali.it (G. Bottaro),g.bottaro93@gmail.com (G. Bottaro), fpalermo@unict.it (F. Palermo) \\ ${ }^{*}$ Corresponding author
}

\section{To cite this article:}

Gaetano Bottaro, Giuseppe Bottaro, Filippo Palermo. Flu and Flu Vaccination in Comparison, in the Evaluation of the Health Status of Children. American Journal of Pediatrics. Vol. 6, No. 3, 2020, pp. 253-258. doi: 10.11648/j.ajp.20200603.23

Received: May 27, 2020; Accepted: June 17, 2020; Published: July 4, 2020

\begin{abstract}
Influence often have severe consequences in the short and long term, and could cause fatal outcomes in some of the sick people. We evaluated the impact of flu vaccines on the health of children in the months following the epidemics, comparing these data with those of children who, on the other hand, caught the flu. The present study was performed between 2014/15 and 2018/19 (5 seasons), focusing on children aged between 6 months and 14 years old, and it compared two group of children, sorted according to whether they received the flu vaccine or contracted flu. We demonstrate, in a statistically significant way, that children who get vaccinated with the seasonal flu vaccine catch fewer diseases in the following months, compared to those who catch the flu, especially with respect to the feared acute otitis media and wheezing. Moreover, vaccinated children receive fewer antibiotic therapies and, consequently, they attend the office of the family pediatrician less. We conclude that flu vaccine protects any child, even if this child is healthy, and does not suffer from any preexisting pathology, during the months after vaccination.
\end{abstract}

Keywords: Influence, Flu Vaccination, Impact on the Health of Children

\section{Introduction}

Influence or flu is an infectious disease affecting the upper and lower respiratory tract, caused by viruses belonging to the Orthomyxoviridae family, divided into three types: A, B and C. Flu is a seasonal disease that usually affects the winter months. Viruses are transmitted through air, by means of saliva drops emitted with coughing and sneezing, and commonly cause fever, cough, sore throat, headache and muscle pain. Generally speaking, the infection has a mild course and regresses within a week, but in rare cases it can cause complications such as otitis, pneumonia, bronchitis, bronchopneumonia and sinusitis, particularly in categories at risk as children [1, 2].

The flu virus is easily transmitted through air, typically by means of inhaling droplets resulting from the coughing or sneezing of infected people. It can also be transmitted through contacts between hands which were previously contaminated with respiratory secretions, and therefore a careful hand hygiene is essential to limit the spread of the disease [3]. Moreover, it is possible to get infected through touching infected surfaces or objects and consequently introducing the virus into the body through touching our own mouth or nose $[4,5]$.

In humans, the flu virus reproduces within the epithelial cells of the respiratory system. Normally, it penetrates a cell, mainly that of the columnar epithelium, causing complex cytopathic effects. Therefore, the loss of essential cellular proteins causes necrosis and death of all infected cells (6). There are numerous individual factors that can determine a certain degree of protection from the virus or that conversely increase the risk of a fatal outcome as the result of a particular type of flu [7], together with genetic factors that are likely to affect this individual susceptibility [8]. 
The main form of prevention against influenza is vaccination $[5,9,10]$. Vaccine stimulates antibody production against flu viruses, even though these latter have the peculiarity of changing their surface proteins' pattern quite often: for this reason, vaccination must be repeated annually, especially for the groups at higher risk, including children affected by chronic diseases increasing the likelihood of developing serious complications.

Flu is a serious public health problem and results into very high human and socio-health costs [11]. Every year, flu affects millions of people, often causing several complications in people at risk, likely with unfavorable outcome. Seasonal flu epidemics are predictable and, most of all, entirely preventable through vaccination, which over the years has proven to be a safe and effective measure [12]. However, despite the safety and protection offered, influenza vaccines are insufficiently used, especially by those health workers and physicians who, on the contrary, should carry them out both for the protection of their patients and to give the good example (Pisa Charter 2017) [13].

Moving to the effects of the flu, we will focus now on those suffered by children. Important epidemiological studies, carried out in the USA [14, 15] and in Spain [16], have clearly shown how, during winter periods, when influenza viruses give rise to classic epidemics, the number of children who get ill with flu-like forms was higher compared to that of seasons without flu. Unfortunately, there are no cohort studies on the correlation between the individual child and the short-term protective effect of the flu vaccine on his or her general state of health in the months after the contraction of the disease.

For this reason, we attempted to study the impact of flu vaccines on the health of children in the months following the epidemics, therefore typically between January and June, and we have compared these data with that of children who, on the other hand, caught the flu.

\section{Materials and Methods}

Our observational and retrospective study was performed at the office of a family pediatrician. The observation involved the comparison between those children who underwent flu vaccination at the pediatrician's office between 2014/15 and 2018/19 (5 seasons), and those children who contracted the flu during the very same seasons.

The flu vaccination at the pediatrician's office is part of the "Flu Vaccination Program" implemented annually by the authorities of the Region of Sicily, by means of the Decree of the Health Department, published on the Official Journal of the Region of Sicily. This Decree issues from the measures asked by the National Vaccinal Prevention Plan of the Italian Ministry of Health and the Permanent State-Regions Conference.

The certainty of the existence of the disease was given by the Reference Laboratory for the Sicily Region, which is part of national network named "Clinical Epidemiology with Cancer Registry of Palermo and Province" - Department of
Central Hospital Services of the A. O. U. P. "P. Giaccone". Molecular typing of viruses was carried out, within an Epidemiological - Molecular Surveillance project of influenza viruses. Virological monitoring covers epidemic interval and surveillance is provided on the territory by means of the contribution of the so-called 'sentinel physicians', such as family pediatricians and general practitioners. The surveillance is carried out yearly involving a standardized procedure which employs medical records for the collection of epidemiological data, biological samples, courier service in charge of transporting biological samples to central laboratory, and viral identification.

Clinical data relating to the period January-June of each year, from 2015 to 2019 were recovered through the management of the computerized medical records available at the designated pediatrician's office. The data analyzed are:

1) age;

2) chronic pathology;

3 ) the type of vaccine used on vaccinated children;

4) the infecting viral strain affecting sick children;

5) how many and which pathologies every child has suffered during the period of observation;

6) the illnesses taken into account were:
a) rhinitis
b) acute otitis media
c) pharyngitis
d) laryngo-tracheitis
e) sinusitis
f) wheezing
g) low respiratory tract infections (LRTI)
h) other illnesses (gastroenteritis, cystitis, febrile exanthems)

7) how many antibiotic therapies were prescribed in the observation period.

The diagnosis of diseases was made according to the US CDC guidelines (17), as follows:

1) Rhinitis: rhinorrhea, cough, sore throat, fever, headache, muscle pains.

2) Acute otitis media: earache, mono or bilateral bulging, redness and hypomotility of the tympanic membrane.

3) Pharyngitis: sore throat, red pharynx, fever, recommended the rapid antigen detection test for GAS, even if negative.

4) Laryngotracheitis: fever, barking cough, laryngotracheal stridor, negative thoracic finding.

5) Sinusitis: sudden onset of high fever, marked rhinorrhea and cough, 7-10 days after rhinitis or common cold.

6) Wheezing: coughing and wheezing.

7) Low respiratory tract infections (LRTI): high fever $\left(<38^{\circ} \mathrm{C}\right)$, persistent cough, dyspnoea, FR around $50 / \mathrm{min}$, oxygen saturation around $95 \%$, thoracic findings.

8) Other pathologies: Gastroenteritis: vomiting, diarrhea with more than 3 discharges of liquid stool. Cystitis: positive urine test for nitrite and leukocyte $>20$ p. c. m. For febrile rashes, the diagnosis was clinical. 


\subsection{Endpoints of the Study}

The primary endpoints were: 1) the evaluation, in the two groups, of the average number and typology of pathologies suffered by each child during the period of observation and 2) how many antibiotic therapies were prescribed on average for each child in the two groups.

The secondary endpoints were: determining the type of pathology presented by each child and whether there are significant differences resulting from different ages or based on the presence of chronic pathologies.

\subsection{Statistical Analysis}

We employed the whole sample of children for statistical analysis. Since we aimed at undertaking a retrospective analysis of computerized clinical data, gathered during the pediatrician's ordinary work routine and collected anonymously, it was not deemed necessary to collect further informed consent, other than the data collected in the course of pediatricians' ordinary work.

The data were entered into an Excel sheet and analyzed with the SPSS statistical program.

We ran descriptive comparisons between the two groups under analysis, therefore reporting data addressing averages, standard deviations and standard error, for the quantitative variables and in terms of absolute frequencies and percentages for the qualitative variables. To detect significant differences in variations in the primary and secondary endpoints, the nonparametric Mann and Whitney test were used for the means while $\mathrm{chi}_{2}$ test were employed for the frequencies.

\section{Results}

\subsection{General Data}

We collected the data of 657 children, among which 347 were vaccinated and 310 contracted flu. All children were within the age range of 6 months and 14 years old and were respectively: 24 in the 6-24 months range, 165 in the $2-5$ years range, 256 in the 5-9 years range and 212 over 9 years. 112 children were observed for the 2014/15 flu season, 122 in 2015/16, 119 in 2016/17, 144 in 2017/18 and 160 in the 2018/19. All these data are summarized in table 1.

Table 1. General clinical record.

\begin{tabular}{llll}
\hline & Vaccinated Children & Sick Children & Total \\
\hline $\begin{array}{l}\text { Total age range } \\
\text { Age range }\end{array}$ & 6 months - 14 years & & 657 \\
6-24 months old & 13 & 11 & \\
2-5 years old & 78 & 87 & 24 \\
5-9 years old & 131 & 125 & 165 \\
over 9 years old & 125 & 87 & 256 \\
TOTAL & & & 212 \\
Chronic children & 103 & 37 & 657 \\
Flu season & & & 140 \\
2014/15 & 44 & 68 & \\
2015/16 & 49 & 73 & 112 \\
2016/17 & 77 & 42 & 122 \\
2017/18 & 68 & 76 & 119 \\
\hline
\end{tabular}

\begin{tabular}{llll}
\hline & Vaccinated Children & Sick Children & Total \\
\hline $2018 / 19$ & 109 & 51 & 160 \\
TOTAL & & & 657 \\
\hline
\end{tabular}

The vaccines employed, supplied by the Epidemiological Service of the ASP 3 of Catania, are respectively Fluarix tetra 2014 used on 44 children, Fluarix tetra 2015 used on 40 children, Vaxigrip 2015 used on 9 children, Fluarix tetra 2016 used on 64 children, Vaxigrip 2016 used on 13 children, Fluarix tetra 2017 used on 37 children, Influvac 2017 used on 31 children, Vaxigrip tetra 2018 used on 109 children.

The isolated viral strains were $\mathrm{A} / \mathrm{H} 3 \mathrm{~N} 2$ in 160 children, A/H1N1pdm09 in 31 children and B in 119 children (Figure 1).

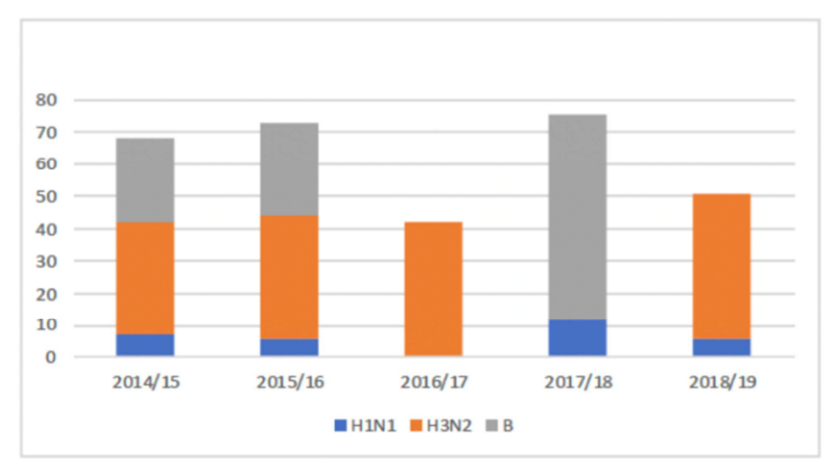

Figure 1. Frequency of viral strains during flu seasons.

Concerning the diseases observed, the most common one was rhinitis with 99 episodes in vaccinated children and 152 in sick ones, followed by acute otitis media with 41 episodes in vaccinated children and 149 in sick ones, then wheezing with 41 episodes in vaccinated children and 101 in sick ones, and finally other pathologies with 30 episodes in vaccinated children and 69 in sick ones. Concerning this first group of diseases, the statistical comparison using $\mathrm{chi}_{2}$ showed high significance $(p<0.001)$. As for the other pathologies, the episodes of pharyngitis were 78 in vaccinated children, while they were 97 in sick ones. The episodes of laryngo-tracheitis were 49 in vaccinated children while they were 44 in sick ones; the episodes of LRTI were 32 in vaccinated children and 14 in sick ones; finally, the episodes of sinusitis were 8 in vaccinated children and 21 in sick ones. In this second group of diseases, despite the numerical differences, these did not show statistically significant differences.

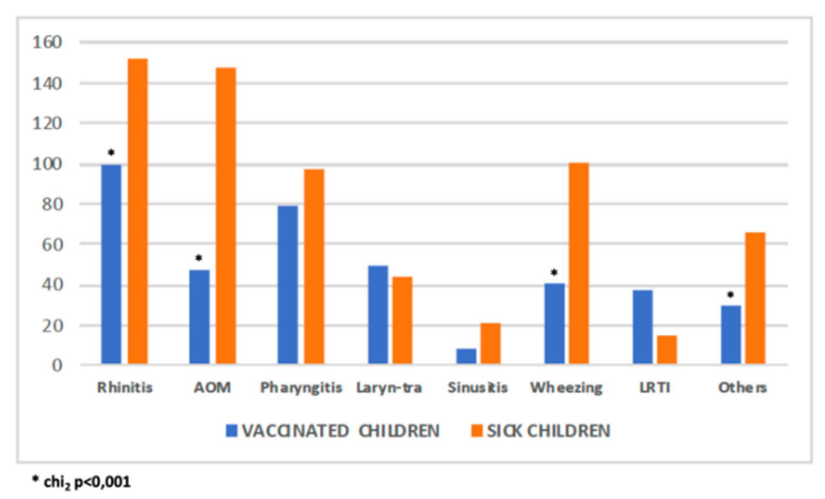

Figure 2. Frequency of the various pathologies in the two groups. 
All the data discussed in this previous paragraph are summarized in figure 2 .

As for the frequency of the diseases, we found an average $1.09 \pm 1.25$ episodes in vaccinated children and $2.09 \pm 1.39$ in sick ones, and the comparison of these averages showed high significance using the Mann and Whitney test (Figure 3). Sick children between 0-24 months-statistically appear to get sick more frequently $(\mathrm{p}<0.001)$ than both vaccinated peers and other sick children (Figure 4).

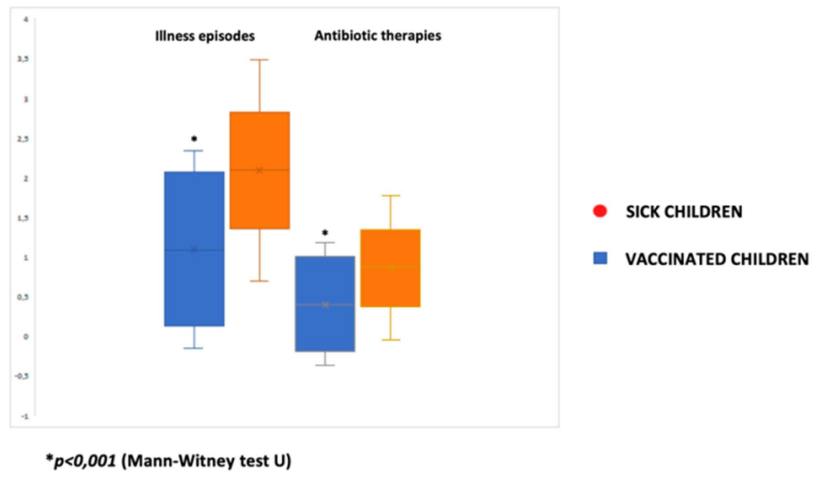

Figure 3. Average of the disease episodes and the antibiotics prescriptions in the two groups.

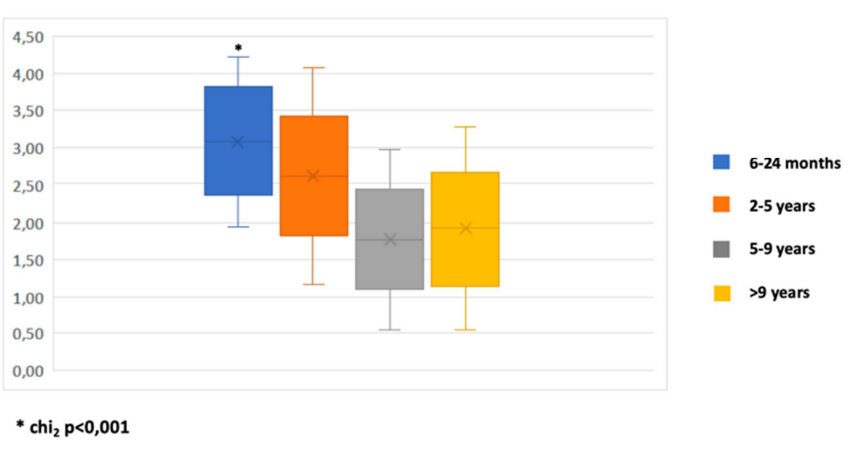

Figure 4. Average of the disease episodes in children with flu, sorted by age.

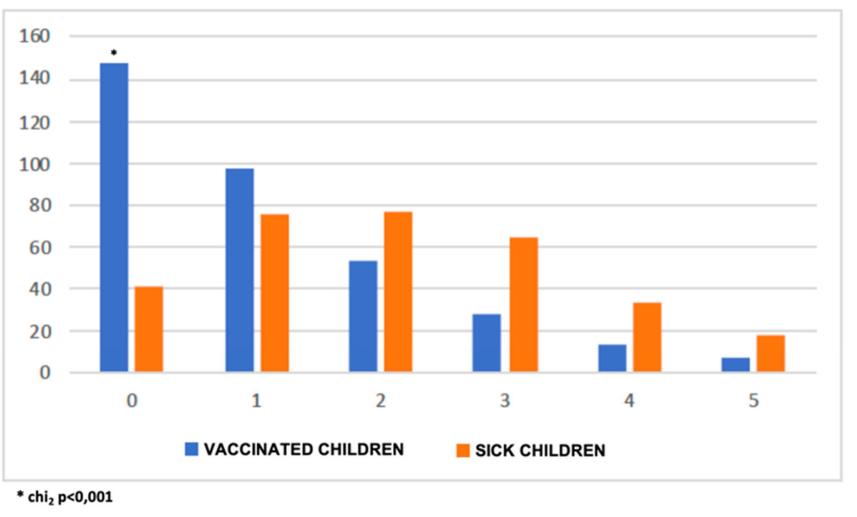

Figure 5. Number of disease events in the two groups.

Moreover, the frequency of episodes of diseases, appears to be of 0 episodes in 147 vaccinated children, while it is of 41 episodes in other ones $\left(\mathrm{chi}_{2} \mathrm{p}<0.001\right), 98$ vaccinated children presented only 1 episode of disease, compared to 76 sick children showing the same frequency and 2 episodes appeared in 77 sick children and only in 53 vaccinated ones. Moreover, 3 episodes appeared in 64 sick children and only in 28 vaccinated ones, 4 episodes appeared in 34 sick children and only in 14 vaccinated ones and, finally, 5 episodes of disease appeared only in 7 vaccinated children and in 18 sick other ones (Figure 5).

Our study concerned as well antibiotic prescriptions, which show the average of $0.40 \pm 0.79$ therapies given to vaccinated children against an average of $0.86 \pm 0.91$ therapies given to sick ones. The comparison between these averages showed high statistical significance $(p<0.001)$ using the Mann and Whitney test (Figure 3).

The frequency of antibiotic prescriptions is of 0 in 254 vaccinated children, and 132 of the sick children (chi2 p $<0.001$ ), while 65 vaccinated children against 107 sick ones received only 1 antibiotic treatment. 2 antibiotic therapies were given to 14 vaccinated children and 58 sick ones, 3 antibiotics therapies were given to 10 vaccinated children and 9 sick ones, while 4 antibiotic therapies were given to 4 vaccinated children and 4 sick other ones.

The main results of the study are summarized in Table 2 .

Table 2. Summary of the data obtained.

\begin{tabular}{llll}
\hline Parameters & $\begin{array}{l}\text { Vaccinate } \\
\text { Children }\end{array}$ & $\begin{array}{l}\text { Sick } \\
\text { Children }\end{array}$ & p \\
\hline Total diseases & 347 & 310 & 0,001 \\
Average of total diseases & 47,2 & 80,9 & 0,001 \\
Average for children & $1,09 \pm 1,25$ & $2,09 \pm 1,39$ & 0,001 \\
Rhynitis & 99 & 152 & 0,001 \\
Acute otitis media & 41 & 149 & 0,001 \\
Pharyngitis & 78 & 97 & \\
Laryngo-tracheitis & 49 & 44 & \\
Sinusitis & 8 & 21 & \\
Wheezing & 41 & 101 & 0,001 \\
Pneumonia-bronchopneumonia & 32 & 14 & \\
Others & 30 & 66 & 0,001 \\
Children with 0 episodes & 147 & 41 & 0,001 \\
Prescripted antibiotics & 93 & 178 & 0,001 \\
Average for children & $0,40 \pm 0,79$ & $0,86 \pm 0,91$ & 0,001 \\
Children without antibiotic prescription & 254 & 132 & 0,001 \\
\hline
\end{tabular}

\subsection{Endpoints of the Study}

The primary endpoints of the study were entirely satisfying. Both the evaluation made on the two groups, namely how many pathologies each child presented on average during the period of observation and how many antibiotic therapies were prescribed on average for each child, resulted into highly significant comparisons according to the Mann and Whitney test for nonparametric data.

The secondary endpoints including the type of pathology presented by each child and the presence of differences based on age and on whether the child's pathology was chronic, gave statistically significant results as well. The only event that did not show statistically significant differences between vaccinated children and those affected by flu was the use of antibiotics in children with chronic diseases. 


\section{Discussion}

Despite certain popular beliefs, influenza is a very dangerous infectious disease. Unfortunately, common feeling has trivialized the term "flu", to the point that today is common to say "I caught the flu" or others similar sentences to indicate suffering from a common cold or a simple congestion. If this habit of keeping a relaxed attitude towards influenza could have been of some use to pastime doctors, trivializing influenza today appears to make little sense. Common feeling often clashes with the real riskiness of the disease, as proven by both the experiences of $2011 \mathrm{H} 1 \mathrm{~N} 1$ and more recently, COVID-19.

Despite all the aforementioned considerations, influenza's vaccine prevention has never really caught on as one would hope partly due to the fact that it must be repeated every year [18], and this is not an issue to be underestimated. [18].

In children, flu can have outcomes that vary from mild to severe ones and, most of all, in children with general health problems and especially with chronic disease (asthma, diabetes, cystic fibrosis, immunodeficiency, nervous system disorders, tumors, obesity, genetic diseases) influenza proves to have high risks of complications such as pneumonia, bronchitis, sinusitis and otitis. However, apart from the short-term complications which are strictly connected to the infection, there is a broader compromise in the general state of health of the child which is not easily detectable, but fortunately does not elude the family pediatrician' attention: the children that caught influenza show a particular sensitivity to sicknesses in the following months [15].

Despite the regrettably low number of studies on the field, it is possible to witness a series of consequences stemming from the use of vaccine: a strong reduction in pediatric deaths from influenza, a clear reduction in early school leaving in vaccinated children a generic reduction of post-flu morbidity in children of all ages [18].

It is specific lack of data that led us to try to collect our owns.

Therefore, we have been able to demonstrate, in a statistically significant way, that children who get vaccinated with the seasonal flu vaccine catch fewer diseases in the following months, then those who catch the flu, especially with respect the feared acute otitis media [19] and wheezing (20). Moreover, these vaccinated children receive fewer antibiotic therapies and, consequently, they attend the office of the family pediatrician less [21]. Our data show as well that the children who benefit the most from vaccines are the youngest-ones, aged 6-24 months.

Considering that the child is believed to be a sort of plague spreader of Manzoninan memory, protecting him or her from an important disease as flu allows to protect the child's family as well, from grandparents to siblings parents, and shields other children in close contact with him or her, from schoolmates to playmates [22].

The only data we were unable to confirm, are those concerning the frequency of LRTI and the amount of antibiotics prescribed to children with chronic pathologies, even though we expect to witness a reduction in numbers concerning vaccinated children. However, these children are those who can make the difference in the statistics. Actually, most of them are included in the vaccinated group, in opposition to that of unvaccinated ones: these children are those who catch more LRTI (we talk about children with cystic fibrosis, immunodeficiency, congenital heart diseases, bronchial asthma) and those who require the highest amount of antibiotics.

The peculiarity of the family pediatrician's job is to collect and keep all the information concerning each child, and to use it during each step of the way. Furthermore, the family pediatrician who believes that flu leads to higher morbidities has the duty to counsel the families and instruct them on how to take greater care of the health of their children.

Vaccinating a child against flu, even if this child is healthy, and does not suffer from any preexisting pathology, proves to be as well an effective way to protect him or her during the months after vaccination. This provides us with a valuable additional weapon of prevention against diseases, the loss of school days, the loss of parents' working days, the excessive use of PS, the excessive hospitalizations and, last but not least, results into a reduction of the workload of family pediatricians.

\section{Conclusions}

We can conclude that flu vaccine protects any child, not only for seasonal flu, but also from all other ILIs during the months after vaccination. This occurs even if this child is healthy, and does not suffer from any preexisting pathology.

\section{Acknowledgements}

We wish to thank Dr. Fabio Tramuto for the kind concession of the data on flu viruses and Dr. Mara Nicosia for the help given in the revision of the text

\section{References}

[1] Bourgeois FT, Valim C, Wei JC, Mc-Adam AJ, Mandl KD. Influenza and other respiratory virus-related emergency department visits among young children. Pediatrics 2006; 118 (1): e1-e8

[2] Molinari NA, Ortega-Sanchez IR, Messonnier ML, Thompson WW, Wortley PM, Weintraub E, Bridges CB. The annual impact of seasonal influenza in the US: measuring disease burden and costs. Vaccine 2007; 25: 5086-96.

[3] Aiello AE, Coulborn RM, Perez V, Larson EL. Effect of hand hygiene on infectious disease risk in the community setting: a meta-analysis. Am J Public Health 2008; 98: 1372-81.

[4] Reed C, Chaves SS, Daily Kirley P, Emerson R, Aragon D, Hancock EB, Butler L, Baumbach J, Hollick G, Bennett NM, Laidler MR, Thomas A, Meltzer MI, Finelli L. Estimating influenza disease burden from population-based surveillance data in the United States. PLoS One 2015; 10: e0118369. 
[5] COMMITTEE ON INFECTIOUS DISEASES Recommendations for Prevention and Control of Influenza in Children, 2019-2020. Pediatrics. 2019 Oct; 144 (4). pii: e20192478. doi: 10.1542/peds.2019-2478. Epub 2019 Sep 2.

[6] Yuen KY, Wong SSY. Human infection by avian influenza A H5N1. Hong Kong Med J 2005; 11: 189-99.

[7] Vaillant L, La Ruche G, Tarantola A et al. Epidemiology of fatal cases associated with pandemic H1N1 influenza 2009. Eurosurveillance 2009; 14: 33.

[8] Trammell RA, Toth LA. Genetic susceptibility and resistance to influenza infection and disease in humans and mice. Expert Rev Mol Diagn. 2008 Jul; 8 (4): 515-29. doi: 10.1586/14737159.8.4.515.

[9] Jain VK, Rivera L, Zaman K, Espos RA, Jr., Sirivichayakul C, Quiambao BP, Rivera-Medina DM, Kerdpanich P, Ceyhan M, Dinleyici EC, Cravioto A, Yunus M, Chanthavanich P, Limkittikul K, Kurugol Z, Alhan E, Caplanusi A, Durviaux S, Boutet P, Ofori-Anyinam O, Chandrasekaran V, Dbaibo G, Innis BL. Vaccine for Prevention of Mild and Moderate-toSevere Influenza in Children. N Engl J Med 2013; 369: 248191.

[10] Grohskopf LA, Sokolow LZ, Broder KR, et al. Prevention and control of seasonal influenza with vaccines. MMWR Recomm Rep 2016; 65: 1-54.

[11] Reed C, Chaves SS, Kirley PD, Emerson P, Aragon D, EB Hancock, Butler L, Baumbach J, Hollick G, Bennett NM, Laidler MR, Thomas A, Meltzer MI, Finelli L. Estimating Influenza Disease Burden from Population-Based Surveillance Data in the United States. PLoS ONE 201510 (3): e0118369. doi: 10.1371/journal.pone.0118369.

[12] Tokars JI, Olsen SJ, Reed C. Seasonal Incidence of Symptomatic Influenza in the United States. CID 2018; 66 (10): 1511-8.

[13] http://www.simpios.eu/wp-content/uploads/2017/06/Carta-diPisa-Vaccinazione-Operatori-Sanitari.pdf.

[14] Flannery B, Reynolds SB, Blanton L, Santibanez TA, O’Halloran A, Peng-Jun Lu, Chen J, Foppa IM, Gargiullo P,
Bresee J, Singleton JA, Fry AM. Influenza Vaccine Effectiveness against Pediatric Deaths: 2010-2014. Pediatrics 139 (5) 2017: e20164244.

[15] Kwong JC, Buchan SA, Chung H, Campitelli MA, Schwartz KL, Crowcroft NS, Jackson ML, Karnauchow T, Katz K, McGeer AJ, McNally JD, Richardson DC, Richardson SE, Rosella LC, Simor A, Smieja M, Zahariadis G, Campigottom A, Gubbay JB. Can routinely collected laboratory and health administrative data be used to assess influenza vaccine effectiveness? Assessing the validity of the Flu and Other Respiratory Viruses Research (FOREVER) Cohort. Vaccine 37 (2019) 4392-4400.

[16] García A, Ortiz de Lejarazu R, Reina J, Callejo D, Cuervo J, Morano Larragueta R. Cost-effectiveness analysis of quadrivalent influenza vaccine in Spain. Hum Vaccin Immunother. 2016 Sep; 12 (9): 2269-77. Epub 2016 May 16.

[17] https://www.cdc.gov/nhsn/pdfs/pscmanual/17pscnosinfdef_cu rrent.pdf.

[18] Esposito S. La prevenzione dell'influenza in età pediatrica. RIAP 2018 (1): 27-34.

[19] Hoberman A, Greenberg DP, Paradise JL, et al. Effectiveness of inactivated influenza vaccine in preventing acute otitis media in young children: a randomized controlled trial. JAMA 2003; 290: 1608-16.

[20] Smits AS, Hak E, Stalman WAB, Van Essen GA, Hoes AW, Verheij JM. Clinical effectiveness of conventional influenza vaccination in asthmatic children. Epidemiol. Infect. (2002), $128,205 \pm 211$.

[21] Pebody RG, Green HK, Andrews N, Boddington NL, Zhao H, Yonova I, Ellis j, Steinberger S, Donati M, Elliot AJ, Uptake and impact of vaccination school age children against influenza during a season with circulation of drifted influenza A and B strain, England 2014/15. Euro Surveill 2015 (39): 111.

[22] Reichert TA, Sugaya N, Fedson DS, Glezen WP, Simonsen L, Tashiro M. The Japanese experience with vaccinating schoolchildren against influenza. N Engl J Med. 2001 Mar 22; 344 (12): 889-96. 